\title{
In vivo anti-tumor activity of photodynamic therapy with intravenous administration of acridine orange, followed by illumination with high-power flash wave light in a mouse osteosarcoma model
}

\author{
HARUHIKO SATONAKA ${ }^{1}$, KATSUYUKI KUSUZAKI ${ }^{2}$, TAKAO MATSUBARA ${ }^{1}$, \\ KEN SHINTANI ${ }^{1}$, TOMOKI NAKAMURA ${ }^{1}$, AKIHIKO MATSUMINE ${ }^{1}$, \\ TAKAHIRO IINO $^{1}$ and ATSUMASA UCHIDA ${ }^{1}$ \\ ${ }^{1}$ Department of Orthopaedic Surgery, Mie University Graduate School of Medicine, Mie; \\ ${ }^{2}$ Research Science Center, Kyoto Prefectural University Graduate School of Medical Science, Kyoto, Japan
}

Received June 24, 2009; Accepted August 25, 2009

DOI: $10.3892 /$ ol_00000012

\begin{abstract}
In a recent study, we demonstrated that a high-power flash wave light (FWL) from a xenon lamp exerted a stronger cytocidal effect against a mouse osteosarcoma cell line than continuous wave light (CWL) in photodynamic therapy with acridine orange (AO-PDT). Based on our in vitro results, we investigated the in vivo anti-tumor activity of AO-PDT using flash wave light from a xenon lamp in a mouse osteosarcoma model. Mouse osteosarcoma cells (LM8) were injected into the subcutaneous tissue of the back of $\mathrm{C} 3 \mathrm{H}$ mice, and tumors that grew to approximately $3 \mathrm{~mm}$ in diameter were treated by AO-PDT using FWL. AO was administered by intravenous injection and $2 \mathrm{~h}$ later the entire body of the mouse was illuminated with FWL from a xenon lamp. Significant growth inhibition of the tumor xenografts was observed as compared with that in the control group, suggesting that AO-PDT with FWL may be useful in the treatment of osteosarcoma. An immunohistochemical study of the tumors treated by AO-PDT showed that the underlying mechanism of the tumor growth inhibition involved both apoptosis and necrosis. In conclusion, it appears that following the intravenous administration of $\mathrm{AO}$, AO-PDT in combination with FWL exerts strong anti-tumor activity. Inhibitory effects against growth of the primary tumor in human patients with osteosarcoma as well as other musculoskeletal sarcomas were also observed.
\end{abstract}

Correspondence to: Dr Katsuyuki Kusuzaki, Research Science Center, Kyoto Prefectural University Graduate School of Medical Science, 465 Kawaramachi Hirokoji Kajiichou, Kamigyou-Ku, Kyoto, Japan

E-mail: kusuzaki@koto.kpu-m.ac.jp

Key words: osteosarcoma, acridine orange, photodynamic therapy, flash wave light, apoptosis

\section{Introduction}

Effort has been devoted to preserving excellent limb function with a low risk of local tumor recurrence after marginal or intralesional tumor resection. Thus, over the past 10 years, we have engaged in the development of photodynamic and radiodynamic therapy with acridine orange (AO-PDT and AO-RDT) as a minimally invasive surgery for the treatment of musculoskeletal sarcomas. Numerous clinical trials for this treatment modality conducted thus far have shown to be successful (1-7). However, in these clinical studies on AO-PDT and AO-RDT, AO was administered by local and not by intravenous injection. Furthermore, AO excitation was achieved using continuous wave light (CWL) derived from a xenon lamp, as opposed to a high-power flash wave light (FWL) from a xenon lamp that has been demonstrated in previous studies to exert a stronger cytocidal effect, as compared to CWL, using a mouse osteosarcoma cell line $(8,9)$. These studies also showed that the intravenous administration of AO may be useful for the photodynamic diagnosis (PDD) of mouse osteosarcoma, without entailing any complications (10). Therefore, using a mouse osteosarcoma model, we undertook to clarify the antitumor activity of AO-PDT with FWL after the intravenous administration of AO.

\section{Materials and methods}

Mouse osteosarcoma model. LM8, the mouse osteosarcoma cell line used in the present study, was derived from Dunn's osteosarcoma which possesses strong metastasizing ability (11). LM8 cells were harvested in Dulbecco's modified Eagle's medium containing $10 \%$ fetal bovine serum at $37^{\circ} \mathrm{C}$ in a $5 \%$ $\mathrm{CO}_{2}$ atmosphere. A suspension containing $1 \times 10^{6}$ cells isolated from the culture dishes by trypsinization was inoculated into the soft tissues, including the subcutaneous tissue and muscles of the back, at the site of implantation in $\mathrm{C} 3 \mathrm{H}$ mice after removal of the hair (5-week-old males; Japan SLC. Inc., Shizuoka, Japan). The subsequent experiments described 
below were conducted on tumors that grew to a macroscopically detectable size ( $>3 \mathrm{~mm}$ in diameter) within 10 days.

Light sources. A xenon lamp was used as the source of the flash wave light (FWL) (12). The illumination machine KFS$30 \mathrm{HJ}$ (Ushio Electric Inc., Tokyo, Japan) was used for the FWL illumination. The FWL light illumination frequency was 60 $\mathrm{Hz}$ and the pulse width was $<1 \mathrm{~ms}$. The energy generated by one-shot illumination with FWL was $15 \mathrm{~J}$ and the illumination level was 1,000,000 lux.

Tumor growth inhibition by AO-PDT using FWL. The tumorbearing mice were divided randomly into 4 groups of 5 mice each: group 1, no treatment $\left(\mathrm{AO}^{-} / \mathrm{L}^{-}\right)$; group 2, illumination with FWL alone for $10 \mathrm{~min}\left(\mathrm{AO}^{-} / 10 \mathrm{~min} \mathrm{FWL}\right)$; group 3, intravenous administration of $\mathrm{AO}$ at $1.0 \mathrm{mg} / \mathrm{kg}$ alone $(1.0 \mathrm{mg} /$ $\left.\mathrm{kg} \mathrm{AO} / \mathrm{L}^{-}\right)$and group 4, intravenous administration of $\mathrm{AO}$ at $1.0 \mathrm{mg} / \mathrm{kg} \mathrm{AO}$ followed by illumination with FWL for $10 \mathrm{~min}(1.0 \mathrm{mg} / \mathrm{kg} \mathrm{AO} / 10 \mathrm{~min} \mathrm{FWL})$. The tumor-bearing mice administered with $\mathrm{AO}$ via the tail vein were exposed to FWL illumination for $10 \mathrm{~min}$ at $2 \mathrm{~h}$ after the AO injection. Fig. 1 shows the system used for the FWL illumination of the mice. Mice administered with AO were set in a stainless steel bowl under anesthesia induced by intraperitoneally administered pentobarbital sodium, and then exposed to FWL illumination from the illumination machine KFS-30HJ for $10 \mathrm{~min}$. The tumor volume was sequentially calculated, until 28 days after this treatment, from the measured values of the maximum and minimum diameters as maximum diameter $\mathrm{x}$ minimum diameter $2 / 2$ (13). AO was used at a concentration of $1.0 \mathrm{mg} / \mathrm{kg}$, since previous studies showed that this concentration yields the strongest cytocidal effect and lowest toxicity in mice (10). The illumination time (10 $\mathrm{min}$ ) and time-point of illumination ( $2 \mathrm{~h}$ after $\mathrm{AO}$ injection) were also selected based on the results of previous studies $(9,10,14,15)$.

Histological and immunohistochemical responses. The histological responses to AO-PDT using FWL were comparatively evaluated in groups 1 and 4 three days after the treatment, using sections of the resected tumors stained with hematoxylin and eosin. Immunohistochemical analysis by TdT-mediated dUTP-biotin nick end-labeling (TUNEL) was also performed in group 4.

Statistical analysis. Statistical analysis was performed using the StatView statistical software (version 5.0; SAS Institute Inc., Cary, NC, USA). Significant differences among the various groups were evaluated using Student's t-test. $\mathrm{P}<0.05$ was considered to be significant.

Experiments were performed in accordance with the guidelines in the Declaration of Helsinki and the Interdisciplinary Principles and Guidelines for the Use of Animals in Research, Testing and Education.

\section{Results}

Tumor growth inhibition by AO-PDT using FWL. A significant decrease in the tumor volume was observed in group 4 $(1.0 \mathrm{mg} / \mathrm{kg} \mathrm{AO} / 10 \mathrm{~min} \mathrm{FWL})$ as compared to that in groups 1 $\left(\mathrm{AO}^{-} / \mathrm{L}^{-}\right)(\mathrm{p}<0.02), 2\left(\mathrm{AO}^{-} / 10 \mathrm{~min} \mathrm{FWL}\right)(\mathrm{p}<0.05)$ or $3(1.0 \mathrm{mg} /$

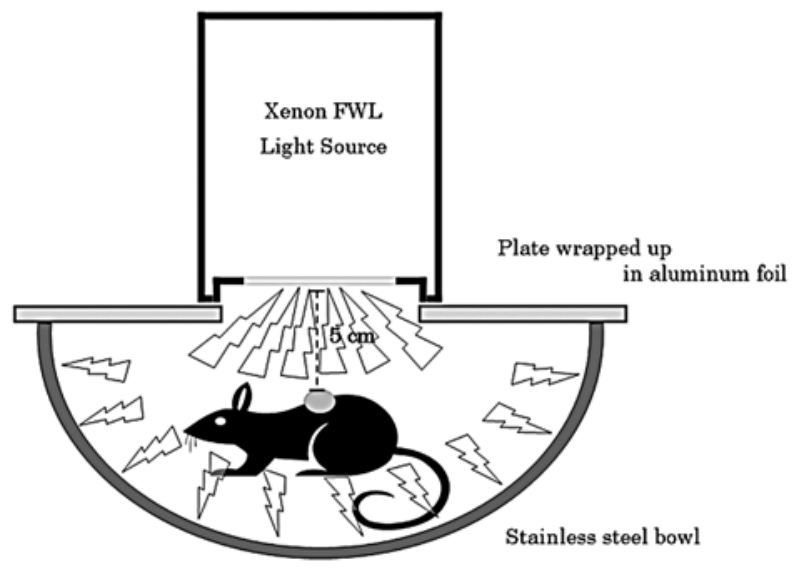

Figure 1. The system of flash wave light (FWL) illumination of tumor-bearing mice is shown.

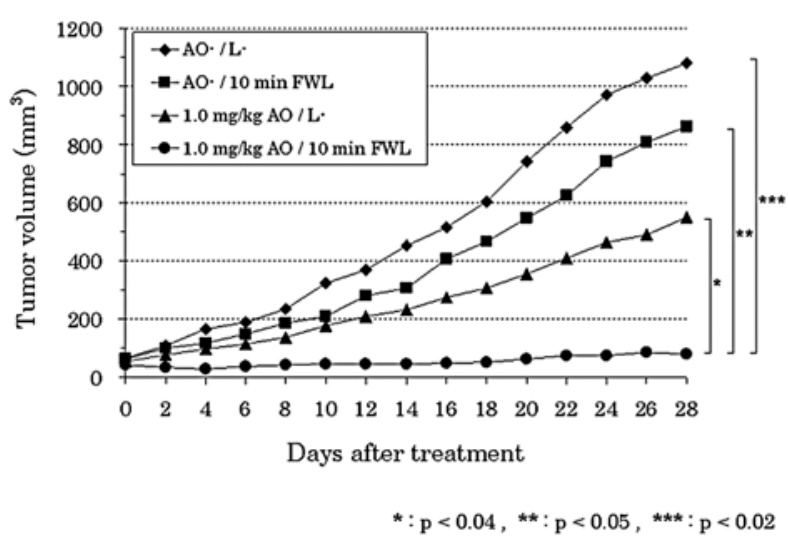

Figure 2. Growth of mouse osteosarcomas after intravenous injection of $\mathrm{AO}$ at $1.0 \mathrm{mg} / \mathrm{kg}$, followed by illumination with FWL. A significant decrease of the tumor volume was noted in group $4(1.0 \mathrm{mg} / \mathrm{kg} \mathrm{AO} / 10 \mathrm{~min} \mathrm{FWL})$ as compared to that in groups $1\left(\mathrm{AO}^{-} / \mathrm{L}^{-}\right)(\mathrm{p}<0.02), 2\left(\mathrm{AO}^{-} / 10 \mathrm{~min} \mathrm{FWL}\right)(\mathrm{p}<0.05)$ and 3 $\left(1.0 \mathrm{mg} / \mathrm{kg} \mathrm{AO} / \mathrm{L}^{-}\right)(\mathrm{p}<0.04)$.

$\left.\mathrm{kg} \mathrm{AO} / \mathrm{L}^{-}\right)(\mathrm{p}<0.04)$. The tumor volume tended to decrease in group 3 as compared to that in groups 1 or 2 . However, there were no significant differences among the three groups (Fig. 2).

Histological and immunohistochemical responses. A histological examination of the tumors showed substantial cell necrosis in group 4, but not in group 1. The surviving tumor cells in group 4 showed large pyknotic nuclei (Fig. 3). Immunohistochemical analysis by TUNEL assay revealed the presence of numerous apoptotic cells (Fig. 4).

\section{Discussion}

Our previous intensive basic investigation and clinical trials showed the strong cytocidal effect of photodynamic and/ or radiodynamic therapy with acridine orange (AO-PDT and AO-RDT). Findings suggest that this type of treatment modality is useful with minimally invasive surgery for patients with high-grade malignant musculoskeletal sarcomas, as it allows for the maintenance of excellent limb function and is correlated with a low risk of local recurrence (3-10,14-19). 


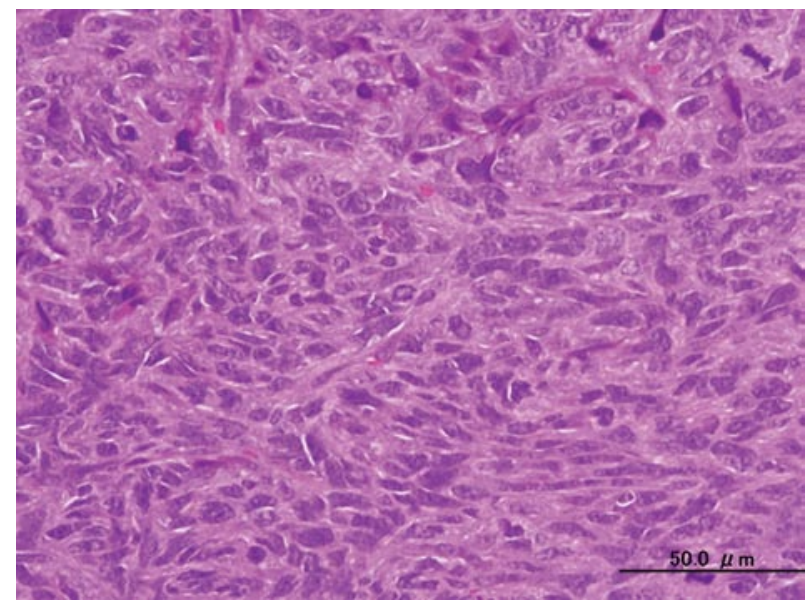

B

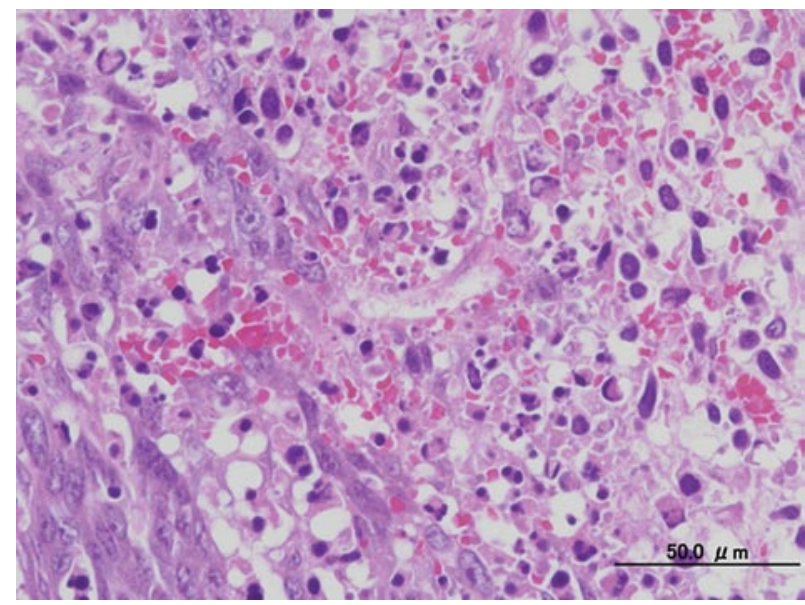

Figure 3. Histological findings in the mouse osteosarcoma xenografts in (A) group $1\left(\mathrm{AO}^{-} / \mathrm{L}^{-}\right)$and $(\mathrm{B})$ group $4(1.0 \mathrm{mg} / \mathrm{kg} \mathrm{AO} / 10 \mathrm{~min} \mathrm{FWL})$, three days after AO-PDT (H\&E stain; original objective lens, $\mathrm{x} 10)$.

However, further improvements in the techniques of this innovative modality are required. At present, in clinical AO-PDT, $\mathrm{AO}$ is administrated locally, not systemically, and any residual tumor after intralesional resection is illuminated by CWL from a xenon lamp. We contended that for the homogeneous uptake of $\mathrm{AO}$ by the entire tumor burden in the human body, intravenous administration may be better than local administration and that stronger excitation with a high-power light may yield a stronger cytocidal effect. To confirm the latter hypothesis, we performed an in vitro study using a mouse osteosarcoma cell line in which AO-PDT was achieved using high-power FWL, which is commonly used for strobe photos and has a stronger illumination at lower energy levels than CWL. After a 10-min illumination and following exposure to $\mathrm{AO}$ at the concentration of $1.0 \mathrm{mg} / \mathrm{ml}$, FWL showed a stronger cytocidal effect than CWL on the mouse osteosarcoma cell line, LM8 (9). Consequently, this in vivo study was conducted. Previously, we reported that the intraperitoneal administration of $\mathrm{AO}$ at $10 \mathrm{mg} /$ $\mathrm{kg}$ followed by blue light excitation inhibited the tumor growth of osteosarcoma developing from a different cell line (MOS) than LM8 in vivo $(14,15,18,19)$. Recently, we also found that the intravenous administration of $\mathrm{AO}$ at $1.0 \mathrm{mg} / \mathrm{kg}$ is useful for PDD of mouse osteosarcomas in nude mice. AO initially binds to both tumor and normal tissues, such as muscles and adipose

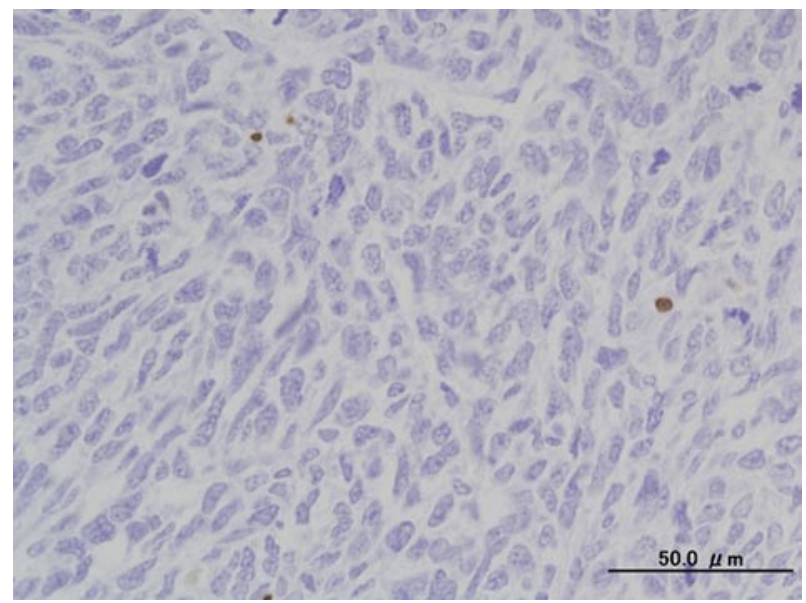

B

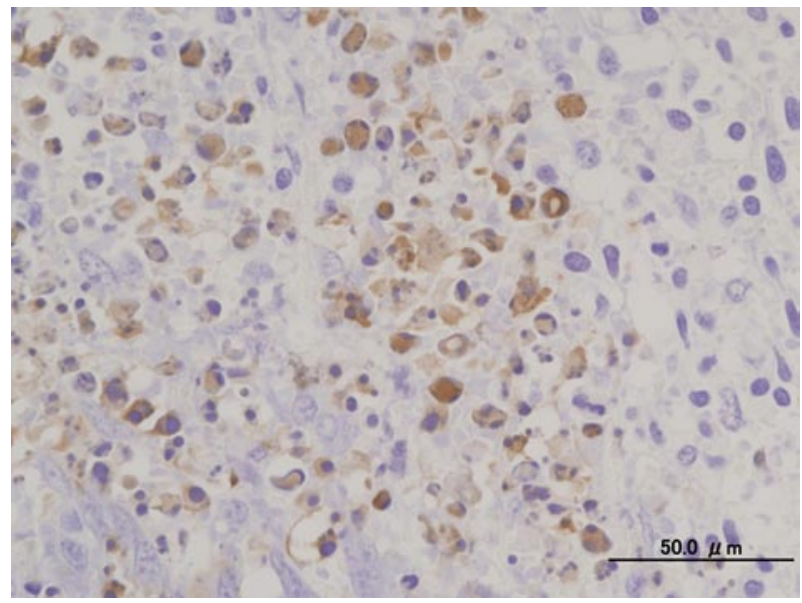

Figure 4. Findings of TUNEL assay in the mouse osteosarcoma xenografts in (A) group $1\left(\mathrm{AO}^{-} / \mathrm{L}^{-}\right)$and (B) group 4 (1.0 mg/kg AO/10 $\left.\mathrm{min} \mathrm{FWL}\right)$, three days after AO-PDT (original objective lens, x10).

tissue. However, after $2 \mathrm{~h} \mathrm{AO}$ is quickly excluded from the normal, but not malignant, tissues. This exclusion produces a significant difference of the AO fluorescence intensity between tumor and normal tissues, thereby allowing visualization of the tumor tissue alone which fluoresces under blue excitation light. In the PDD study, we determined that the LD50 of the intravenously administered AO was $27.3 \mathrm{mg} / \mathrm{kg}$ and that none of the mice showed fetal complications after administration of the compound at a dose of $<27.3 \mathrm{mg} / \mathrm{kg}$. Therefore, AO at the concentration of $1.0 \mathrm{mg} / \mathrm{kg}$ should be safe for humans (10).

The findings of this study showed that the growth of mouse osteosarcoma tumors was significantly inhibited in the group treated with AO-PDT using FWL following the intravenous administration of $\mathrm{AO}$, as compared with that in the control groups, including the non-treatment, AO-alone and FWL-alone treatment groups. Histological and immunohistochemical examinations on day 3, following AO-PDT, showed that apoptosis and necrosis were induced in the tumor cells $(20,21)$, although we have yet to clarify the precise genetic pathway leading to cell death induced by AO-PDT. None of the mice treated with AO-PDT died. Therefore, we hypothesize that AO-PDT, with AO intravenously administered at $1.0 \mathrm{mg} / \mathrm{kg}$, followed by excitation using FWL, shows cytocidal effects against osteosarcoma cells. As demonstrated 
in our previous in vitro study, the use of FWL for AO-PDT yields a stronger cytocidal effect than that of CWL (9). Since the results showed that the intravenous administration of $\mathrm{AO}$ at $1.0 \mathrm{mg} / \mathrm{kg}$ is useful for AO-PDT and is safe for mice, AO may also be applicable in humans, although intensive clinical studies are needed to confirm the efficacy and toxicity of AO administered by intravenous injection.

In conclusion, AO-PDT using FWL, following the intravenous administration of $\mathrm{AO}$, exerted strong anti-tumor activity against the primary tumor. Consequently, this treatment modality is applicable for the treatment of sarcomas as well as carcinomas in humans, and in future, will be employed as an innovative modality for cancer therapy.

\section{References}

1. Dougherty TJ and Marcus SL: Photodynamic therapy. Eur J Cancer 28A: 1734-1742, 1992.

2. Moan J and Berg K: Photochemotherapy of cancer: experimental research. Photochem Photobiol 55: 931-948, 1992.

3. Kusuzaki K, Murata H, Matsubara T, et al: Clinical trial of photodynamic therapy using acridine orange with/without low dose radiation as new limb salvage modality in musculoskeletal sarcomas. Anticancer Res 25: 1225-1236, 2005.

4. Kusuzaki K, Murata H, Matsubara T, et al: Clinical outcome of a novel photodynamic therapy technique using acridine orange for synovial sarcomas. Photochem Photobiol 81: 705-709, 2005.

5. Yoshida K, Kusuzaki K, Matsubara T, et al: Periosteal Ewing's sarcoma treated by photodynamic therapy with acridine orange. Oncol Rep 13: 279-282, 2005.

6. Kusuzaki K, Murata H, Matsubara T, Satonaka H, Wakabayashi T, Matsumine A and Uchida A: Acridine orange could be an innovative anticancer agent under photon energy (Review). In Vivo 21: 205-214, 2007.

7. Nakamura T, Kusuzaki K, Matsubara T, Matsumine A, Murata $\mathrm{H}$ and Uchida A: A new limb salvage surgery in cases of high-grade soft tissue sarcoma using photodynamic surgery, followed by photo- and radiodynamic therapy with acridine orange. J Surg Oncol 97: 523-528, 2008.

8. Ueda H, Murata H, Takeshita H, Minami G, Hashiguchi S and Kubo T: Unfiltered xenon light is useful for photodynamic therapy with acridine orange. Anticancer Res 25: 3979-3984, 2005.
9. Satonaka H, Kusuzaki K, Matsubara T, et al: Flash wave light strongly enhanced the cytocidal effect of photodynamic therapy with acridine orange on a mouse osteosarcoma cell line. Anticancer Res 27: 3339-3344, 2007.

10. Satonaka H, Kusuzaki K, Matsubara T, Shintani K, Wakabayashi T, Matsumine A and Uchida A: Extracorporeal photodynamic image detection of mouse osteosarcoma in soft tissues utilizing fluorovisualization effect of acridine orange. Oncology 70: 465-473, 2006.

11. Asai T, Ueda T, Itoh K, Yoshioka K, Aoki Y, Mori S and Yoshikawa $\mathrm{H}$ : Establishment and characterization of a murine osteosarcoma cell line (LM8) with high metastatic potential to the lung. Int J Cancer 76: 418-422, 1998.

12. Kimura M, Kashikura K, Yokoi S, Koiwa Y, Tokuoka Y and Kawashima N: Photodynamic therapy for cancer cells using a flash wave light xenon lamp. Opt Rev 12: 207-210, 2005.

13. Ovejera AA, Houchens DP and Barker AD: Chemotherapy of human tumor xenografts in genetically athymic mice. Ann Clin Lab Sci 8: 50-56, 1978.

14. Kusuzaki K, Suginoshita T, Minami G, et al: Fluorovisualization effect of acridine orange on mouse osteosarcoma. Anticancer Res 20: 3019-3024, 2000.

15. Kusuzaki K, Minami G, Takeshita H, et al: Photodynamic inactivation with acridine orange on a multidrug-resistant mouse osteosarcoma cell line. Jpn J Cancer Res 91: 439-445, 2000.

16. Matsubara T, Kusuzaki K, Matsumine A, Shintani K, Satonaka H and Uchida A: Acridine orange used for photodynamic therapy accumulates in malignant musculoskeletal tumors depending on pH gradient. Anticancer Res 26: 187-194, 2006.

17. Kusuzaki K, Murata H, Takeshita $\mathrm{H}$, et al: Intracellular binding sites of acridine orange in living osteosarcoma cells. Anticancer Res 20: 971-976, 2000.

18. Kusuzaki K, Aomori K, Suginoshita T, et al: Total tumor cell elimination with minimum damage to normal tissues in musculoskeletal sarcomas following photodynamic therapy with acridine orange. Oncology 59: 174-180, 2000.

19. Hashiguchi S, Kusuzaki K, Murata H, et al: Acridine orange excited by low-dose radiation has a strong cytocidal effect on mouse osteosarcoma. Oncology 65: 85-93, 2005.

20. Kessel D and Luo Y: Mitochondrial photodamage and PDTinduced apoptosis. Photochem Photobiol 42: 89-85, 1998.

21. Zdolsek JM, Olson M and Brunk UT: Photooxidative damage to lysosomes of cultured macrophages by acridine orange. Photochem Photobiol 51: 67-76, 1990. 